\title{
CONSUMO DE ALIMENTO BALANCEADO EN REEMPLAZOS DE LECHERÍA DESDE EL NACIMIENTO HASTA LAS OCHO SEMANAS DE EDAD
}

\author{
Jorge Alberto Elizondo-Salazar ${ }^{\otimes}$, Cynthia Rebeca Monge-Rojas ${ }^{2}$
}

\section{RESUMEN}

Las terneras nacen con un rumen sin desarrollarse física y metabólicamente, lo que las clasifica como pre-rumiantes que dependen de la leche para obtener los nutrientes necesarios para crecer. Estimular el consumo de materia seca se convierte por lo tanto en una parte vital en la alimentación de terneras con el fin de llenar sus requerimientos de crecimiento y a la vez estimular el desarrollo del rumen desde temprano y así convertirse en un rumiante funcional. El consumo adecuado de materia seca es el principal objetivo de cualquier programa de alimentación ya que de este depende que los animales adquieran los nutrientes necesarios para desarrollar su potencial productivo, por lo que es importante conocer la concentración de energía de la dieta, la digestibilidad de la ración, palatabilidad, procesamiento, ambiente y consumo de agua, con el fin de estimular el consumo en los animales. Debido a que en Costa Rica existen pocas referencias de los niveles de consumo o ganancia de peso diarios para los animales en la etapa pre-destete, este trabajo pretende analizar algunas de las investigaciones realizadas en los últimos años con el fin de determinar el consumo de alimento en la etapa de predeste. Después de analizar cinco estudios en condiciones similares se encontraron variaciones en el consumo de alimento balanceado a la semana ocho, donde osciló entre 527 y $1017 \mathrm{~g}$, con ganancias de peso que variaron desde los 513 hasta los $714 \mathrm{~g} / \mathrm{d}$. Las variaciones en los consumos se pueden deber a algunas diferencias en el desarrollo de las investigaciones, factores raza o la variabilidad entre individuos por lo que es necesario desarrollar más investigaciones para lograr establecer datos de referencia bajo las condiciones del país.

Palabras clave: Ganado de leche, crianza de terneras, nutrición animal, nutrientes, consumo de alimento.

\footnotetext{
'Inscrito en la Vicerrectoría de Investigación de la Universidad de Costa Rica. Proyecto 737-B4-222.

${ }^{2}$ Universidad de Costa Rica. Facultad de Ciencias Agroalimentarias, Estación Experimental Alfredo Volio Mata. Ochomogo, Cartago.

Autores para correspondencia: jorge.elizondosalazar@ucr.ac.cr (https://orcid.org/0000-0003-26039635), cynthia.mongerojas@ucr.ac.cr

Recibido: 20 setiembre 2019 Aceptado: 08 noviembre 2019

Esta obra está bajo licencia Internacional Creative Commons

https://creativecommons.org/licenses/by-nc-nd/4.0/
} 


\section{ABSTRACT}

Dairy replacements feed consumption from birth to eight weeks of age. Calves are born with a physical and metabolically undeveloped rumen that classifies them as preruminants that depend on the milk to get the necessary nutrients to grow. Stimulating the consumption of dry matter then becomes a vital part in the feeding of calves in order to meet their growth requirements and at the same time stimulate the development of the rumen as soon as possible and thus become a functional ruminant. Dry matter consumption is the main objective of any feeding program since it depends on the animals to consume the necessary nutrients to develop their potential, so it is very important to be aware of the energy concentration of the diet, ration digestibility, palatability, processing, environment and water consumption, in order to stimulate consumption in animals. In Costa Rica there are few references to daily consumption or weight gain for animals in the pre-weaning stage; this work consists of analyzing some of the research carried out in recent years in order to determine the ideal consumption level. After analyzing five studies under similar conditions, variations in consumption were found at week eight, where the consumption of concentrate ranged from 527 to $1017 \mathrm{~g}$, with weight gains ranging from 513 to $714 \mathrm{~g} / \mathrm{d}$. The variations in consumption may be due to some differences in research methodology, breed or variability between individuals, so it is necessary to carry out more research to achieve reference data under our country's conditions.

Key words: Dairy cattle, calf rearing, animal nutrition, nutrients, feed intake. 


\section{INTRODUCCIÓN}

Las terneras y novillas, también conocidas como animales en crecimiento o reemplazos son el futuro de la producción en toda finca lechera, por lo que cuidar de ellas se vuelve una prioridad, con el fin de que estos animales logren llegar al primer servicio entre los 14 y 16 meses de edad y así den inicio a su ciclo productivo. Uno de los mayores retos fisiológicos en las primeras semanas de vida de la ternera son los eventos alrededor del desarrollo del rumen, por lo que es de suma importancia asegurarse que los animales reciban una alimentación apropiada desde los primeros días de vida y que logren mantener consumos adecuados con el fin de garantizar un desarrollo adecuado del mismo.

Debido a la importancia que representa la etapa de crecimiento de las terneras, el consumo de materia seca se convierte en un factor clave en su desarrollo, ya que determina la cantidad de nutrientes disponibles y está ligado en gran parte con los rendimientos productivos del mismo, por lo que se puede decir que es el parámetro más crítico en un programa de alimentación y puede verse afectado por variables como la concentración de energía en la dieta, factores ambientales y de manejo, entre otros (NRC, 2001).

El destete es una etapa particularmente importante en la vida de las terneras, implica un cambio en el manejo y la alimentación, y si no se brinda el cuidado adecuado pueden presentar un retroceso en las primeras semanas post-destete, y un retraso en el crecimiento, debido a que enfrentan un cambio dietético muy fuerte especialmente si a la hora del destete no han llegado al adecuado consumo de materia seca y desarrollo ruminal (van Ackeren et al., 2009). Es por esta razón que el desarrollo físico y metabólico del retículo rumen en las terneras jóvenes es un pre requisito para la transición de la dieta líquida a la dieta sólida, ya que el desarrollo del rumen está determinado principalmente por la cantidad y naturaleza del alimento sólido ofrecido, debido a que el desarrollo del epitelio ruminal es estimulado por la producción de ácidos grasos volátiles (especialmente butírico y propiónico) resultados de la fermentación microbiana de los carbohidratos solubles que ingresan al rumen, mientras que el desarrollo de la musculatura del rumen, los movimientos ruminales y su capacidad son influenciados por el estímulo físico proveniente del material fibroso consumido (Nemati et al., 2015). 
El objetivo del presente trabajo es resaltar la importancia del consumo de materia seca en las terneras desde edades tempranas y presentar algunos datos de investigaciones que se han realizado en nuestro país.

\section{Consumo de materia seca}

El consumo de alimento está regulado por una serie de señales, en las que el cerebro, específicamente el hipotálamo y regiones aledañas, recogen información de sensores especiales y receptores ubicados en la pared del tracto digestivo y los tejidos que forman parte en el metabolismo. Estos receptores transmiten su información vía nervio vago y el sistema nervioso simpático, para que luego esta información sea integrada y utilizada con el fin de determinar que se necesita comer y si el consumo debe iniciar o terminar (Forbes, 1996; Fisher, 2002).

También hay otros reguladores involucrados en el consumo de materia seca entre los que se deben considerar la distensión, capacidad del rumen, concentración de proteína y energía y sus interrelaciones. Además, es importante tener en cuenta que los aspectos de comportamiento también influyen sobre el consumo voluntario a través de asociaciones resultado de una retroalimentación vía post-ingesta, ya que se ha comprobado que los rumiantes pueden identificar alimentos particulares y modificar el consumo de acuerdo con experiencias pasadas (Forbes, 1980; Forbes, 1996; Fisher, 2002).

El consumo de materia seca en los rumiantes jóvenes debe iniciarse desde la primera semana de edad, ya que es vital para que la ternera pase de ser un animal pre rumiante a un rumiante completamente funcional (Coverdale et al., 2004). Se ha demostrado que existe también una relación directa entre el consumo de alimento, el consumo de agua y la ganancia de peso de los animales (Kertz et al., 1984; Monge-Rojas y Elizondo-Salazar, 2016a). También es importante recalcar que a pesar de que el consumo de forraje promueve el desarrollo muscular del rumen, estimula la rumia y el flujo de saliva dentro del rumen, las dietas basadas en granos han demostrado mayores consumos de materia seca que aquellas basadas solamente en forrajes (van Ackeren et al., 2009). 
El manejo pre destete convencional donde se le suministra a los animales alrededor de un $10 \%$ de su peso corporal como leche o reemplazador lácteo, es el resultado de numerosas investigaciones que establecen esa cantidad de leche como el ideal para conseguir que los animales consuman hasta el doble de alimento que aquellos con acceso a mayores cantidades de leche, y es que a pesar de mejorar las tasas de crecimiento, la eficiencia alimenticia y disminuir la incidencia de enfermedades y los signos evidentes de hambre, un mayor consumo de leche impacta el consumo de alimento sólido y por ende retrasa el desarrollo ruminal (Khan et al., 2007; Sweeney, et al., 2010; Khan et al., 2011a; Khan et al., 2011b; Elizondo-Salazar y Sánchez-Alvarez, 2012; Stamey et al., 2012). Esta reducción de consumo de alimento sólido cuando los animales tienen mayores suministros de leche, se debe a que las terneras no tienen tanta hambre debido al sentimiento de saciedad provocado por el llenado químico y mecánico.

Se ha observado que una vez que lo animales comienzan con el consumo de alimento sólido realmente parecen necesitar consumirlo ya que en su ausencia lo buscan y hasta se han visto comiendo el material de las camas, lo que sugiere la necesidad del consumo de materiales sólidos conforme crecen (Díaz et al., 2001), siempre tratando que ese alimento sólido sea alimento balanceado y no forraje pues este puede evitar el consumo de pre iniciador y por ende cambiar la fermentación en el rumen produciendo acetato en lugar de butirato y propionato, retrasando así el desarrollo papilar en el rumen (Coverdale et al., 2004; Khan et al., 2011b), como se indicó anteriormente.

Es importante tener en cuenta que el procesamiento y forma física del alimento impactan la palatabilidad y el consumo en las terneras. Así, por ejemplo, el peletizado mejora la palatabilidad además de reducir la segregación de ingredientes en los iniciadores y aumenta la digestibilidad, lo que al final puede impactar el consumo de materia seca (Terré et al., 2015; Pazoki et al., 2017). Sin embargo, Suarez-Mena et al. (2015) encontraron que alimentos peletizados no afectaron el desarrollo de las papilas del rumen o la digestibilidad de la dieta.

Una vez que los animales se destetan, el consumo de materia seca va a ser gobernado por la capacidad digestiva (volumen del rumen, patrones de fermentación del alimento, absorción y actividades metabólicas del epitelio del rumen, flujo de la ingesta y motilidad ruminal), los atributos físico-químicos del alimento sólido (naturaleza de los carbohidratos, procesamiento de los alimentos, concentración y calidad de la proteína, tamaño de partícula) así como de cambios en los metabolitos post-absorción por lo que es de suma Nutrición Animal Tropical 13(2): 58-75. ISSN: 2215-3527/ 2019 
importancia que el rumen haya tenido una excelente estimulación durante el pre-destete (Khan et al., 2011a).

\section{Desarrollo del rumen}

En sus primeros meses de vida las terneras viven como monogástricos ya que al nacimiento los primeros tres compartimientos de su estómago (rumen, retículo y omaso) aún no se han desarrollado y no participan en el proceso digestivo. Una vez que las terneras empiezan a consumir alimento balanceado y agua, el rumen comienza a desarrollarse por lo que el consumo de materia seca juega un papel muy importante, no solo en el desarrollo y crecimiento del animal sino en el desarrollo físico y metabólico de lo que será la cámara fermentativa que permitirá al animal aprovechar los alimentos fibrosos al máximo (van Ackeren et al., 2009; Wickramasinghe et al., 2019).

El desarrollo físico del rumen se puede subdividir en dos aspectos: el incremento de la masa y el desarrollo de las papilas. A pesar de que el rumen tiene una habilidad innata de desarrollarse con el tiempo, las dietas líquidas basadas en leche han demostrado retrasar su desarrollo ruminal ya que, en el pre-rumiante los líquidos pasan directamente al abomaso por medio de la escotadura esofágica sobrepasando el rumen, mientras que el alimento balanceado se fermenta en el rumen contribuyendo así con el desarrollo de este (Coverdale et al., 2004; Lesmeister y Heinrichs, 2004; van Ackeren et al., 2009; Laarman y Oba 2011; Elizondo-Salazar y Sánchez-Álvarez, 2012; Nemati et al., 2015; Schäff et al., 2018).

Los ácidos grasos volátiles proveen cerca del $70 \%$ de los requerimientos de energía de los rumiantes. Los tres ácidos grasos volátiles más abundantes producidos en el rumen son el acetato, propionato y butirato. El acetato se necesita primordialmente para energía periférica y en la vaca adulta una parte es incorporada en la grasa de la leche. EI propionato es usado para producir glucosa para energía en el hígado. Sin embargo, el rol del butirato es muy diferente. Más del $80 \%$ del butirato producido en el rumen es metabolizado a cuerpos cetónicos (betahidroxibutirato y acetoacetato) antes de ser transportados al resto del cuerpo para suplir necesidades energéticas. También tiene un destino alternativo de convertirlo directamente en energía aprovechable en el rumen, y por lo tanto es de gran importancia para el desarrollo ruminal (Baldwin et al., 2004). 
La presentación física del alimento también podría tener influencia sobre el desarrollo del rumen, ya que, la alteración de la forma física y/o química durante el procesamiento de los alimentos incrementa el área de absorción o aprovechamiento de los nutrientes. Sin embargo, Castro-Flores y Elizondo-Salazar (2012) al evaluar el efecto del procesamiento del alimento balanceado utilizado en la crianza de terneras, sobre el crecimiento de los animales y el desarrollo ruminal durante el período pre-destete, encontraron que animales que consumieron alimento extrusado presentaron un menor desarrollo de las papilas y menor grosor de la pared ruminal $(P<0,05)$ al compararlo con animales que consumieron alimento en harina. El largo de las papilas no solo es la variable más influenciable por la dieta consumida sino que es la más importante en la evaluación del desarrollo ruminal y se ve afectada positivamente por la producción de butirato que se relaciona con la alteración física de los alimentos por lo que el molido o quebrado de los granos va a tener mayor efecto sobre el desarrollo papilar que los procesos térmicos que producen más propionato en la fermentación ruminal (Lesmeister et al., 2004; Khan et al., 2008).

\section{Requerimientos nutricionales y su relación con el consumo}

Los requerimientos nutricionales de energía (Cuadro 1) y proteína dictados por el NRC (2001) pretenden maximizar la salud y el crecimiento de las terneras, pero es importante recalcar que para lograrlo se requiere de numerosos factores incluyendo el manejo del calostro al nacimiento, el adecuado manejo sanitario y desde luego la alimentación (Rauba et al., 2019). Sin embargo, con la amplia variedad de reemplazadores de leche y alimentos tipo iniciador que existen en el mercado, es de vital importancia comprender la relación entre los requerimientos de proteína y energía de los animales. Se han realizado múltiples investigaciones a lo largo del tiempo con el fin de evaluar las concentraciones de proteína y energía suministradas en la dieta y su relación con el crecimiento de las terneras (Sejrsen y Purup, 1997; Kertz y Loften, 2013; De Paula et al., 2017). 
Cuadro 1. Requerimientos de energía para terneras de lechería.

\begin{tabular}{|c|c|c|c|c|c|c|c|c|}
\hline \multirow[b]{2}{*}{ Peso vivo $(\mathrm{kg})$} & \multirow[b]{2}{*}{ GDP (kg) } & \multicolumn{7}{|c|}{ Energía (Mcal) } \\
\hline & & $\mathrm{EM}_{\mathrm{m}}$ & $\mathrm{EM}_{\mathrm{g}}$ & $\mathrm{EM}_{\mathrm{t}}$ & $\mathrm{EN}_{\mathrm{m}}$ & $\mathrm{EN}_{\mathrm{g}}$ & $\mathrm{EN}_{\mathrm{t}}$ & ED \\
\hline \multirow[t]{3}{*}{20} & 0,00 & 0,95 & 0,00 & 0,95 & 0,81 & 0,00 & 0,81 & 0,99 \\
\hline & 0,20 & 0,95 & 0,35 & 1,30 & 0,81 & 0,24 & 1,06 & 1,35 \\
\hline & 0,40 & 0,95 & 0,81 & 1,76 & 0,81 & 0,56 & 1,37 & 1,83 \\
\hline \multirow[t]{3}{*}{25} & 0,00 & 1,12 & 0,00 & 1,12 & 0,96 & 0,00 & 0,96 & 1,16 \\
\hline & 0,20 & 1,12 & 0,38 & 1,50 & 0,96 & 0,26 & 1,22 & 1,56 \\
\hline & 0,40 & 1,12 & 0,88 & 2,00 & 0,96 & 0,61 & 1,57 & 2,08 \\
\hline \multirow[t]{3}{*}{30} & 0,00 & 1,28 & 0,00 & 1,28 & 1,10 & 0,00 & 1,10 & 1,34 \\
\hline & 0,20 & 1,28 & 0,41 & 1,69 & 1,10 & 0,28 & 1,38 & 1,76 \\
\hline & 0,40 & 1,28 & 0,94 & 2,22 & 1,10 & 0,65 & 1,75 & 2,31 \\
\hline \multirow[t]{3}{*}{35} & 0,00 & 1,44 & 0,00 & 1,44 & 1,24 & 0,00 & 1,24 & 1,50 \\
\hline & 0,20 & 1,44 & 0,43 & 1,87 & 1,24 & 0,30 & 1,53 & 1,95 \\
\hline & 0,40 & 1,44 & 0,99 & 2,43 & 1,24 & 0,68 & 1,92 & 2,53 \\
\hline \multirow[t]{4}{*}{40} & 0,00 & 1,59 & 0,00 & 1,59 & 1,37 & 0,00 & 1,37 & 1,66 \\
\hline & 0,20 & 1,59 & 0,45 & 2,04 & 1,37 & 0,31 & 1,68 & 2,13 \\
\hline & 0,40 & 1,59 & 1,04 & 2,63 & 1,37 & 0,72 & 2,08 & 2,74 \\
\hline & 0,60 & 1,59 & 1,69 & 3,28 & 1,37 & 1,16 & 2,53 & 3,41 \\
\hline \multirow[t]{4}{*}{45} & 0,00 & 1,74 & 0,00 & 1,74 & 1,49 & 0,00 & 1,49 & 1,81 \\
\hline & 0,20 & 1,74 & 0,47 & 2,21 & 1,49 & 0,32 & 1,82 & 2,30 \\
\hline & 0,40 & 1,74 & 1,08 & 2,82 & 1,49 & 0,75 & 2,24 & 2,94 \\
\hline & 0,60 & 1,74 & 1,76 & 3,50 & 1,49 & 1,21 & 2,71 & 3,64 \\
\hline \multirow[t]{4}{*}{50} & 0,00 & 1,88 & 0,00 & 1,88 & 1,62 & 0,00 & 1,62 & 1,96 \\
\hline & 0,20 & 1,88 & 0,49 & 2,37 & 1,62 & 0,34 & 1,95 & 2,47 \\
\hline & 0,40 & 1,88 & 1,12 & 3,00 & 1,62 & 0,77 & 2,39 & 3,13 \\
\hline & 0,60 & 1,88 & 1,82 & 3,71 & 1,62 & 1,26 & 2,88 & 3,86 \\
\hline \multirow[t]{4}{*}{60} & 0,00 & 2,16 & 0,00 & 2,16 & 1,85 & 0,00 & 1,85 & 2,25 \\
\hline & 0,20 & 2,16 & 0,52 & 2,68 & 1,85 & 0,36 & 2,21 & 2,79 \\
\hline & 0,40 & 2,16 & 1,20 & 3,35 & 1,85 & 0,83 & 2,68 & 3,49 \\
\hline & 0,60 & 2,16 & 1,95 & 4,10 & 1,85 & 1,34 & 3,20 & 4,27 \\
\hline
\end{tabular}


En una revisión de literatura, Kertz y Loften (2013) demostraron que utilizar reemplazadores de leche con más de $20 \%$ de proteína cruda es beneficioso para que las terneras obtengan un adecuado crecimiento de tejido magro. Sejrsen y Purup (1997) encontraron que alimentar a las terneras con excesos de grasa podía causar detrimentos en el desarrollo, entre otros de la glándula mamaria, mientras que dietas con contenidos altos de energía y proteína producen mayores ganancias de peso y mejoran la eficiencia alimenticia (Rauba et al., 2019).

Es importante también tener en cuenta que una dieta formulada para satisfacer los requerimientos nutricionales no va a lograr el adecuado crecimiento y desarrollo de las terneras si no viene acompañada de acceso a agua fresca y limpia a libre voluntad ya que a pesar de que los requerimientos de agua no han sido adecuadamente descritos (NRC, 2001), su ausencia puede provocar problemas en las funciones corporales y por ende un bajo desempeño de los animales (Kertz et al., 1984; Huuskonen et al., 2011; Wickramsinghe et al., 2019).

\section{Investigaciones en consumo de alimento balanceado realizadas en Costa Rica.}

Entre las investigaciones desarrolladas en Costa Rica en los últimos siete años que evaluaron el consumo de materia seca en terneras desde el nacimiento hasta las ocho semanas de edad y su impacto sobre el crecimiento y ganancia de peso, se encuentran Castro-Flores y Elizondo-Salazar (2012), Elizondo-Salazar y Sánchez-Álvarez (2012), Vargas-Ramírez y Elizondo-Salazar (2014), Monge-Rojas y Elizondo-Salazar (2016b) y Monge-Rojas y Elizondo-Salazar (2017). Todas las investigaciones siguieron el patrón de crianza de terneras común en el país, asegurándose de ofrecer una cantidad adecuada de calostro de buena calidad a los animales en las primeras 4 horas de vida. El alimento sólido se introdujo en la dieta de los animales en la primera semana de vida utilizando un pre iniciador comercial que junto con el suministro de leche (4 L por día) suplía los requerimientos nutricionales de las terneras en esta etapa de vida.

En 2012, Castro-Flores y Elizondo-Salazar evaluaron el efecto del procesamiento del alimento sobre el crecimiento y desarrollo ruminal en 20 terneros Holstein con un peso promedio de $38,08 \mathrm{~kg}$ a los 2-3 días de edad. Los terneros se asignaron aleatoriamente a uno de los 4 tratamientos y fueron alojados en jaulas individuales. Los tratamientos que se utilizaron fueron alimento en harina, alimento en harina+forraje, alimento peletizado y 
alimento extrusado. El alimento balanceado fue formulado para contener igual concentración de nutrientes. Los investigadores encontraron diferencias significativas $(\mathrm{P}<$ 0,05) en el consumo de alimento peletizado en la semana 3 con $0,900 \mathrm{~kg}$ de MS vs 0,793, 0,567, y 0,802 para los tratamientos harina, harina+forraje y extrusado, y del alimento en harina en las últimas tres semanas de experimento $(5,70 ; 6,40$ y $7,03 \mathrm{~kg}$ para las semanas $6,7$ y 8$)$.

Las diferencias en el consumo de alimento no se vieron reflejadas en la ganancia de peso, ya que los pesos no presentaron diferencias $(P>0,05)$ entre tratamientos, el desarrollo ruminal se vio afectado significativamente $(P<0,05)$ por el procesamiento siendo los animales que consumieron alimento extrusado los que presentaron menor grosor en la pared ruminal y menor altura de las papilas. En dicho estudio se esperaba que los animales que consumían alimento extrusado presentaran un mayor desarrollo y crecimiento, pues el beneficio de la extrusión es un aumento de la digestibilidad y disponibilidad de nutrimentos para los animales.

Elizondo-Salazar y Sánchez-Álvarez (2012), en su análisis del efecto de consumo de dieta líquida y alimento balanceado sobre el crecimiento y desarrollo ruminal en 16 terneras y 4 terneros de la raza Holstein que se incluyeron en dos tratamientos: convencional-dieta líquida de forma restringida (4 L, dos veces al día) y el intensivo donde se ofreció la dieta líquida en mayores cantidades hasta alcanzar $8 \mathrm{~L}$ al día (4 $\mathrm{L}$ en dos tomas). Los investigadores encontraron que los animales que consumieron mayores cantidades de leche (hasta $8 \mathrm{~L} / \mathrm{d})$ tuvieron significativamente $(P>0,05)$ menores consumos de alimento 2,56 y $4,14 \mathrm{~kg}$ vs 4,92 y $6,70 \mathrm{~kg}$ para las semanas 7 y 8 respectivamente), así como un menor desarrollo ruminal y crecimiento papilar, aunque no encontraron diferencias significativas $(P>0,05)$ en las ganancias de peso.

Vargas-Ramírez y Elizondo-Salazar (2014) para establecer el costo económico asociado a la cría de reemplazos desde el nacimiento hasta el destete en una finca lechera comercial, determinaron el consumo de alimento y agua relacionándolos con el crecimiento en 23 terneras Holstein de los 0 a 4 meses de edad y encontraron un promedio diario de consumo de alimento de 0,43 kg para la semana 4 y 1,02 kg para la semana 8 , con consumos de agua de $0,72 \mathrm{~L}$ y $2,10 \mathrm{~L}$ respectivamente y con ganancias de peso de 0,21 $\mathrm{Kg}$ para la semana 4 y $0,51 \mathrm{Kg}$ para la semana 8 . Los autores presentaron los datos con el fin de brindar una referencia a técnicos y productores de lo que se puede esperar en las 
primeras semanas de vida de los animales, aunque si cabe mencionar que sus datos estuvieron por debajo de los reportados por otros autores como Coverdale et al. (2004) que reportaron consumos diarios de materia seca de $0,26 \mathrm{~kg}$ en la etapa predestete y $1,89 \mathrm{~kg}$ en la etapa post destete.

Monge-Rojas y Elizondo-Salazar (2016b) evaluaron el efecto del consumo de agua sobre la ingesta de alimento balanceado y los parámetros de crecimiento en 20 terneras de la raza Jersey desde el nacimiento hasta las ocho semanas de edad. Las terneras fueron asignadas aleatoriamente a dos tratamientos: agua a libre consumo y agua restringida, donde los animales en ambos tratamientos tuvieron acceso a alimento balanceado a libre voluntad. No se encontraron diferencias significativas $(P>0,05)$ entre los animales que consumieron agua y los que tenían el acceso restringido al agua. A las ocho semanas de edad las terneras estaban consumiendo en promedio 0,56 kg de MS y 2,53 L de agua por día. En este estudio se esperaba que las terneras con acceso al agua tuvieran mayores consumos de alimento balanceado y por ende un crecimiento superior.

En el 2017, los mismos autores, con el objetivo de evaluar el efecto que tiene el alojamiento individual o en parejas sobre el consumo de alimento balanceado y los parámetros de crecimiento en 20 terneras de la raza Jersey desde el nacimiento hasta las doce semanas de edad, no encontraron diferencias significativas $(P>0,05)$ en los consumos diarios de materia seca $(0,054$ y $0,53 \mathrm{~kg}$ para alojamiento individual y en parejas, respectivamente) a la semana ocho de edad. En dicho estudio, se esperaba que las terneras alojadas en parejas, hubieran presentado mayores consumos de alimento y mayores ganancias de peso, ya que el alojar a las terneras en parejas, les permite interactuar con sus pares desde pequeñas y motiva a las más lentas a aprender a consumir alimento balanceado al observar a sus compañeras, pueden aprender de sus compañeras; además, al permanecer con compañía, son menos propensas a presentar decaimiento (De Paula Vieira et al., 2010; Costa et al., 2015).

Los consumos reportados en las investigaciones antes mencionadas se resumen en el Cuadro 2. En la mayoría de los estudios no se alcanzaron los consumos reportados por la literatura donde para la semana ocho de edad los animales deberían estar consumiendo más de 1,0 kg por día de iniciador (Kertz et al., 1984; Sweeney et al., 2010; Terré et al., 2015), los únicos que reportaron consumos diarios de más de 1,0 kg fueron VargasRamírez y Elizondo-Salazar (2012). Variaciones en los consumos pueden estar relacionadas con las diferencias de peso al nacimiento, las condiciones ambientales y de Nutrición Animal Tropical 13(2): 58-75. ISSN: 2215-3527/ 2019 
manejo así como las materias primas utilizadas en la elaboración de los concentrados y la palatabilidad, que influyen directamente sobre el consumo de materia seca por parte de los animales.

Cuadro 2. Consumo de alimento balanceado diario y acumulado semanal en las primeras ocho semanas de vida de terneras de lechería.

\begin{tabular}{|c|c|c|c|c|c|c|c|c|c|c|}
\hline \multirow{3}{*}{ 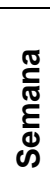 } & \multicolumn{10}{|c|}{ Consumo de alimento, g de MS } \\
\hline & \multicolumn{2}{|c|}{ CF y ES (2012) } & \multicolumn{2}{|c|}{ VR y ES (2012) } & \multicolumn{2}{|c|}{ ES y SA (2014) } & \multicolumn{2}{|c|}{ MR y ES (2016b) } & \multicolumn{2}{|c|}{ MR y ES (2017) } \\
\hline & Diario & Semanal & Diario & Semanal & Diario & Semanal & Diario & Semanal & Diario & Semanal \\
\hline 1 & 28 & 195 & 49 & 343 & 21 & 147 & 19 & 132 & 7 & 52 \\
\hline 2 & 60 & 420 & 173 & 1,209 & 50 & 347 & 43 & 302 & 17 & 116 \\
\hline 3 & 129 & 900 & 285 & 1,993 & 175 & 1,225 & 76 & 534 & 47 & 329 \\
\hline 4 & 189 & 1,322 & 432 & 3,021 & 202 & 1,415 & 153 & 1,068 & 77 & 540 \\
\hline 5 & 495 & 3,467 & 535 & 3,746 & 337 & 2,359 & 213 & 1,489 & 173 & 1,208 \\
\hline 6 & 581 & 4,067 & 745 & 5,214 & 500 & 3,499 & 321 & 2,248 & 281 & 1,970 \\
\hline 7 & 710 & 4,967 & 860 & 6,023 & 703 & 4,924 & 469 & 3,285 & 419 & 2,934 \\
\hline 8 & 837 & 5,856 & 1,017 & 7,117 & 957 & 6,701 & 559 & 3,912 & 527 & 3,687 \\
\hline
\end{tabular}

Los pesos y ganancia diaria de peso de los animales utilizados en los experimentos citados anteriormente se resumen en el Cuadro 3. Para el caso de los dos experimentos realizados con terneras de la raza Jersey reportados por Monge-Rojas y Elizondo-Salazar (2016b y 2017), las terneras doblaron su peso a nacimiento para las ocho semanas de edad. El doblar el peso al nacimiento a las ocho semanas de edad, se considera una medida de eficiencia y adecuado manejo en la crianza de reemplazos. 
Cuadro 3. Peso (kg) y ganancia diaria de peso (GDP, g/d) en terneras de lechería durante las primeras ocho semanas de vida.

\begin{tabular}{ccccccccccc}
\hline \multirow{2}{*}{ Semana } & \multicolumn{2}{c}{$\begin{array}{c}\text { CF y ES } \\
(\mathbf{2 0 1 2})\end{array}$} & \multicolumn{2}{c}{$\begin{array}{c}c \\
\text { (2012) }\end{array}$} & \multicolumn{2}{c}{ ES y SA } & \multicolumn{2}{c}{ MR y ES } & \multicolumn{2}{c}{ MR y ES } \\
\cline { 2 - 10 } & Peso & GPD & Peso & GPD & Peso & GPD & Peso & GPD & Peso & GPD \\
\hline 1 & 35 & - & 41 & - & 48 & - & 27 & - & 28 & - \\
2 & 36 & 142 & 42 & 145 & 50 & 285 & 30 & 470 & 32 & 600 \\
3 & 37 & 142 & 43 & 278 & 51 & 143 & 33 & 460 & 35 & 500 \\
4 & 41 & 571 & 45 & 314 & 52 & 143 & 37 & 440 & 39 & 540 \\
5 & 42 & 142 & 48 & 210 & 54 & 285 & 30 & 490 & 43 & 510 \\
6 & 47 & 714 & 51 & 530 & 55 & 143 & 43 & 510 & 47 & 580 \\
7 & 52 & 714 & 54 & 800 & 57 & 285 & 48 & 640 & 52 & 690 \\
8 & 56 & 571 & 58 & 513 & 62 & 714 & 53 & 650 & 56 & 680 \\
\hline
\end{tabular}

CF-ES (2012): Castro-Flores y Elizondo-Salazar (2012), VR y ES (2012): Vargas-Ramírez y Elizondo-Salazar (2012), ES y SA (2014): Elizondo-Salazar y Sánchez-Álvarez (2014), MR y ES (2016b): Monge-Rojas y Elizondo-Salazar (2016b), MR y ES (2017) Monge-Rojas y Elizondo-Salazar (2017)

En las investigaciones consideradas, el consumo diario de materia seca varió de 527 a 1017 g por día, por lo que resulta muy difícil poder establecer valores estándar de consumo de materia seca en la etapa de pre-destete, especialmente al considerar la gran variabilidad que puede existir entre individuos. Las estrategias para promover el desarrollo de la estructura morfológica y la función metabólica del rumen en los pre-rumiantes son un problema continuo que atrae mucho la atención de la comunidad científica. Numerosos estudios y enfoques intentan modular la fermentación ruminal y la comunidad microbiana en rumiantes jóvenes para acelerar el desarrollo del rumen. Estos enfoques incluyen la alteración de la composición de la dieta y las formas físicas, la adición de nuevos tipos de aditivos para alimentos balanceados y la introducción de variables en el manejo de la alimentación. 
Los productores y productoras deben utilizar estrategias que motiven a las terneras a consumir alimento balanceado de buena calidad y palatabilidad desde muy temprana edad y proveer de agua fresca y limpia todos los días. Es importante destacar también que un consumo de $1,0 \mathrm{~kg}$ de MS por día a las ocho semanas de edad, puede ser suficiente para obtener un adecuado desarrollo ruminal, pero puede que no sea suficiente para llenar los requerimientos nutricionales que garanticen un adecuado crecimiento y desarrollo físico de los animales, especialmente cuando se consideran razas grandes como la Holstein. Por lo tanto, además de considerar el consumo de materia seca, se debe tener en cuenta el consumo de nutrientes, especialmente de energía y proteína.

\section{CONSIDERACIONES FINALES}

Los remplazos de lechería son la piedra angular de cualquier producción; sin embargo, en muchas ocasiones estos animales son olvidados o no se les presta la atención adecuada ya que se considera una etapa improductiva, olvidando que es una etapa determinante en estos animales ya que el cuidado y alimentación que se les dé en este tiempo determinará su futuro desempeño productivo y reproductivo, por lo que conocer el consumo de alimento en esta etapa de crecimiento que supla los requerimientos nutricionales para un crecimiento y desarrollo adecuado, y que a su vez estimule al desarrollo del rumen, se vuelve vital.

Es importante tener en cuenta que el consumo de materia seca se ve afectado por un gran número de factores y en edades tempranas principalmente antes del destete este va a influir directamente en el desarrollo del rumen que determinará la eficiencia productiva del animal, debido a que si el rumen no se encuentra bien desarrollado los animales no serán capaces de aprovechar al máximo los forrajes que se convertirán en el mayor componente de su ración como adultos. 


\section{LITERATURA CITADA}

Baldwin R.L., K.R., McLeod, J.L. Klotz, and R.N. Heitmann. 2004. Rumen development, intestinal growth and hepatic metabolism in the pre- and postweaning ruminant. J. Dairy Sci. 87(Suppl. E): E55-E65.

Castro-Flores, P., y J.A. Elizondo-Salazar. 2012. Crecimiento y desarrollo ruminal en terneros alimentados con iniciador sometido a diferentes procesos. Agronomía Mesoamericana. 23 (2): 343-352.

Costa, J.H.C., R.K. Meagher, M.A.G. Von Keyserlingk, and D.M. Weary. 2015. Early pair housing increases solid feed intake and weight gains in dairy calves. J. Dairy Sci. 98: 1-6.

Coverdale, J.A., H.D. Tyler, J.D. Quigley, and J.A. Brumm. 2004. Effect of various levels of forage and form of diet on rumen development and growth in calves. J. Dairy Sci. 87: 2554-2562.

De Paula, M.R., C.E. Oltramari, J.T. Silva, M.P.C. Gallo, G.B. Mourão, and C.M.M. Bittar. 2017. Intensive liquid feeding of dairy calves with a medium crude protein milk replacer: Effect on performance, rumen, and blood parameters. J. Dairy Sci. 100: 4448-4456.

De Paula Vieira, A., M.A.G. Von Keyserlingk., and D.M. Weary. 2010. Effects of pair versus single housing on performance and behavior of dairy calves before and after weaning form milk. J. Dairy Sci. 93: 3079-3085.

Diaz, M.C., E.E. Van Amburg, J.M. Smith, J.M. Kelsey, and E.L. Hutten. 2001. Composition of growth of Holstein calves fed milk replacer from birth to 105kilograms body weight. J. Dairy Sci. 84: 830-842.

Elizondo-Salazar, J.A., y M. Sánchez-Álvarez. 2012. Efecto del consumo de dieta líquida y alimento balanceado sobre el crecimiento y desarrollo ruminal en terneras de lechería. Agronomía Costarricense. 36 (2): 81-90.

Fisher, D.S. 2002. A review of a few key factors regulating voluntary feed intake in ruminants. Crop Sci. 42: 1651-1655.

Forbes, J.M. 1980. Physiological aspects of the regulation of food intake. Ann. Zootech. 29: 189-196.

Forbes, J.M. 1996. Integration of regulatory signals controlling forage intake in ruminants. J. Anim. Sci. 74: 3029-3035. 
Huuskonen, A., L. Tuomisto, and R. Kauppinen. 2011. Effect of drinking water temperature on water intake and performance of dairy calves. J. Dairy Sci. 94: 2475-2480.

Kertz, A.F., L.F. Reutzel, and J.H. Mahoney. 1984. Ad libitum water intake by neonatal calves and its relationship to calf starter intake, weight gain, feces score and season. J. Dairy Sci. 67: 2964-2969.

Kertz, A.F., and J.R. Loften. 2013. Review: A historical perspective of specific milkreplacer feeding programs in the United States and effects on eventual performance of Holstein dairy calves. Prof. Anim. Sci. 29: 321-332.

Khan, M., H. Lee, W. Lee, H. Kim, K. Ki, T. Hur, G. Suh, S. Kang, and Y. Choi. 2007. Structural growth, rumen development, and metabolic and immune responses of Holstein male calves fed milk through step-down and conventional methods. J. Dairy Sci. 90:3376-3387.

Khan, M., H. Lee, H. Kim, S. Kim, K. Ki, S. Park, J. Ha, and Y. Choi. 2008. Starch source evaluation in calf starter: II. Ruminal parameters, rumen development, nutrient digestibilities, and nitrogen utilization in Holstein calves. J. Dairy Sci. 91: 11401149.

Khan, M.A., D.M. Weary, and M.A.G. von Keyserlingk. 2011a. Invited review: Effects of milk ration on solid feed intake, weaning and performance in dairy heifers. J. Dairy Sci. 94: 1071-1081.

Khan, M.A., D.M. Weary, and M.A.G. von Keyserlingk. 2011b. Hay intake improves performance and rumen development of calves fed higher quantities of milk. J. Dairy Sci. 94: 3547-3553.

Laarman, A.H., and M. Oba. 2011. Effect of calf starter on rumen pH of Holstein dairy calves at weaning. J. Dairy Sci. 94: 5661-5664.

Lesmeister, K.E., and A.J. Heinrichs. 2004. Effects of corn processing on growth characteristics, rumen development, and rumen parameters in neonatal dairy calves. J. Dairy Sci. 87: 3439-3450.

Monge-Rojas, C.R. y J.A. Elizondo-Salazar. 2016a. La importancia del agua en la crianza de remplazos de lechería. Nutrición Animal Tropical. 10(1):1-9.

Monge-Rojas, C.R. y J.A. Elizondo-Salazar. 2016b. El consumo de agua y su efecto sobre la ingesta de alimento balanceado y el crecimiento en terneras Jersey. Nutrición Animal Tropical. 10(2): 75-90. 
Monge-Rojas, C.R. y J.A. Elizondo-Salazar. 2017. Alojamiento individual o en parejas y su efecto sobre el consumo de alimento balanceado y el crecimiento en terneras Jersey. Nutrición Animal Tropical. 11(1): 38-51.

Nemati, M., H. Amanlou, M. Khorvash, B. Moshiri, M. Mirzaei, M.A., Khan, and M.H. Ghaffari. 2015. Rumen fermentation, blood metabolites, and growth performance of calves during transition from liquid to solid feed: Effects of dietary level and particle size of alfalfa hay. J. Dairy Sci. 98: 7131-7141.

NRC (National Research Council). 2001. Nutrient requirements of dairy cattle. $7^{\text {th }}$ rev. ed. Natl. Acad. Press, Washington, DC.

Pazoki, A., G.R. Ghorbani, S. Kargar, A. Sadeghi-Sefidmazgi, J.K. Drackley, and M.H. Ghaffari. 2017. Growth performance, nutrient digestibility, ruminal fermentation, and rumen development of calves during transition from liquid to solid feed: Effects of physical form of starter feed and forage provision. Anim. Feed Sci. Technol. 234: 173-185.

Rauba, J., B.J. Heins, H. Chester-Jones, H.L. Diaz, D. Ziegler, J. Linn, and N. Broadwater. 2019. Relationships between protein and energy consumed form milk replacer ad starter intake and calf growth and firs-lactation production of Holstein dairy cows. J. Dairy Sci. 102: 301-310.

Schäff, C.T., J. Gruse, J. Maciej, R. Pfuhl, R. Zitnan, M. Rajsky, and H.M. Hammon. 2018. Effects of feeding unlimited amounts of milk replacer for the first 5 weeks of age on rumen and small intestinal growth and development in dairy calves. J. Dairy Sci. 101: 783-793.

Sejrsen, K., and S. Purup. 1997. Influence of prepuberal feeding level on milk yield potential of dairy heifers: a review. J. Anim. Sci. 75: 828-835.

Stamey, J.A., N.A. Janovick, A.F. Kertz, and J.K. Drackley. 2012. Influence of starter protein content on growth of dairy calves in an enhanced early nutrition program. J. Dairy Sci. 95: 3327-3336.

Suarez-Mena, F.X., A.J. Heinrichs, C.M. Jones, T.M. Hill, and J.D. Quigley. 2015. Digestive development in neonatal dairy calves with either whole or ground oats in the calf starter. J. Dairy Sci. 98: 3417-3431.

Sweeney, B.C., J. Rushen, D.M. Weary, and A.M. de Pasillé. 2010. Duration of weaning, starter intake, and weight gain of dairy calves fed large amounts of milk. J. Dairy Sci. 93: 148-152. 
Terré, M., L.I. Castells, M.A. Khan, and A. Bach. 2015. Interaction between the physical form of the starter feed and straw provision on growth performance of Holstein calves. J. Dairy Sci. 98: 1101-1109.

van Ackeren, C., H. Steinngaß, K. Hartung, R. Funk, and W. Drochner. 2009. Effect of roughage level in a total mixed ration on feed intake, ruminal fermentation patterns and chewing activity of early-weaned calves with ad libitum access to grass hay. Anim. Feed Sci. Technol. 153: 48-59.

Vargas-Ramírez, A.M., y J.A. Elizondo-Salazar. 2014. Determinación de consumo de alimento balanceado y agua, y medidas de crecimiento en terneras Holstein en una finca lechera comercial. Nutrición Animal Tropical. 8(2): 36-50.

Wickramasinghe, H.K.J.P., A.J. Kramer, and J.A.D.R.N. Appuhamy. 2019. Drinking water intake of newborn dairy calves and its effects on feed intake, growth performance, health status, and nutrient digestibility. J. Dairy Sci. 102: 377-387. 